\title{
LA SATISFACCIÓN DEL CLIENTE Y SU INCIDENCIA EN LA FIDELIZACIÓN DE CLIENTES, EN LAS EMPRESAS DE HOSPEDAJE NO CLASIFICADAS DE LA LOCALIDAD DE HUANCAYO - PERÚ
}

Autor:

Bernaldo Sabuco, L.

\section{RESUMEN}

En la presente investigación de tipo aplicada y nivel explicativo, partiendo del objetivo general: DETERMINAR LA INCIDENCIA DE LA SATISFACCIÓN DEL CLIENTE EN LA FIDELIZACIÓN DE LAS EMPRESAS DE HOSPEDAJE DE LA LOCALIDAD DE HUANCAYO y siguiendo los procedimientos del método científico y otros específicos como el inductivo-deductivo y el analítico-sintético, además de la aplicación de una encuesta a una muestra de 170 clientes extraída de 67 centros de hospedaje de la localidad se ha determinado que la satisfacción del cliente incide de manera positiva y significativa en la fidelización de las empresas de hospedaje de la localidad de Huancayo con un $95 \%$ de confianza. La fuerza de la incidencia es del $63,6 \%$ lo cual se considera como alta. Dentro de la variable independiente se estudiaron independientemente los atributos tangibilidad, fiabilidad, capacidad de respuesta, profesionalismo, cortesía, seguridad, credibilidad/accesibilidad y comprensión con el cliente; por otro lado dentro de la variable dependiente se estudió el atributo fidelización; finalmente se relacionaron ambas variables se concluyó que existe fuerte relación entre ambas.

Palabras Clave: Satisfacción, cliente, fidelización.

\section{SUMMARY:}

In the present investigation applied type and level of explanation, based on the overall objective: DETERMINE THE IMPACT OF CUSTOMER SATISFACTION IN THE FIDELITY OF HOSTING COMPANIES IN THE TOWN OF HUANCAYO and following the procedures of the scientific method and other specifics as the inductive -deductive and analytic-synthetic, as well as the implementation of a survey to a sample of 170 clients drawn from 67 centers hosting the town has determined that customer satisfaction affects positively and significantly on the loyalty of business hosting of the town of Huancayo with $95 \%$ confidence. The strength of the incidence is $63.6 \%$ which is considered high. Within the independent variable attributes were studied independently tangibility, reliability, responsiveness, professionalism, courtesy, security, reliability / availability and customer understanding, on the other hand within the dependent variable studied the loyalty attribute and finally were related both variables was concluded that there is strong relationship b e $t w$ e e $n$

$\mathrm{t} h \mathrm{e}$ $t w o$.

Keywords: satisfaction, customer loyalty.

\section{INTRODUCCIÓN}

En lo que atañe a este trabajo de investigación 
debo declarar de primera intención, que en el proceso de estudio se ha recurrido a distintos investigadores y autores de bibliografía especializada, de quienes sus trabajos han sido mis benévolos amigos que contribuyeron a darle consistencia a este esfuerzo, sobre todo para la elaboración del marco teórico y conceptual que sirvió para orientar la investigación. Esto resulta ineludible cuando se trabaja sobre un tópico en el que han laborado muchas de las mentes más esclarecidas de la humanidad; sin embargo, es preciso aclarar, que con respecto a la incidencia de la satisfacción del cliente en la fidelización de clientes de las empresas de hospedaje (no clasificadas) de la localidad de Huancayo, no se encontraron antecedentes concretos de estudios realizados. En todo caso este trabajo es en respuesta a una complicada problemática de la localidad de Huancayo, debido a que múltiples problemas ponen en riesgo la viabilidad de las empresas de hospedaje, en el mediano y largo plazo; problemas que podemos enumerar, entre otros, a la ausencia de financiamiento a costo competitivo para emprender nuevos proyectos en una economía de ámbito, la elevada presión tributaria, la competencia basada en precios, el inadecuado manejo de las ventas debido a la sobredemanda del servicio hotelero, la baja concurrencia de clientes procedentes del extranjero, la deficiente capacitación de los recursos humanos, la poca presencia del estado con programas de desarrollo del sector hotelero vinculado al desarrollo turístico y de negocios, así como la ausencia de esfuerzos de los empresarios para lograr la satisfacción de los clientes. Entre otros, son estos problemas los que ponen en serio riesgo la rentabilidad de estas MYPE; posiblemente el más importante y el problema que orientó este trabajo, tiene origen interno en las empresas, nos referimos al bajo nivel de satisfacción del cliente ya que tiene efectos directamente sobre el grado de fidelización de clientes y éste a su vez en la rentabilidad del negocio. Son pocos los hospedajes que se preocupan por que sus clientes retornen tan pronto como puedan, conforme a sus cronogramas de viaje; más bien, están preocupados por la rentabilidad inmediata que le brinda la visita de los usuarios del servicio. A falta de alojamiento adecuado muchos visitantes dejan de viajar a la ciudad Huancayo afectando la rentabilidad de los transportistas y taxistas, salas de cinematografía y conferencias, restaurantes, etc., toda una cadena de negocios en riesgo. En este sentido para el estudio se formuló, como problema general, la siguiente interrogante: ¿De qué manera la satisfacción del cliente incide en la fidelización de clientes en las empresas de hospedaje (no clasificadas) de la localidad de Huancayo?; asimismo, los problemas específicos se formularon con las siguientes interrogantes ¿Cuál es el grado de satisfacción de los huéspedes de los hospedajes (no clasificados) de la localidad Huancayo, en cuanto a los atributos "tangibles" del servicio de habitaciones?, ¿Cuál es el grado de satisfacción de los huéspedes de los hospedajes (no clasificados) de la localidad Huancayo, en cuanto al atributo "fiabilidad" en el servicio de habitaciones?, ¿Cuál es el grado de satisfacción de los huéspedes de los hospedajes (no clasificados) de la localidad Huancayo, en cuanto al atributo "capacidad de respuesta" en el servicio de habitaciones?, ¿Cuál es el grado de satisfacción de los huéspedes (no clasificados) de los hospedajes de la localidad Huancayo, en cuanto al atributo "profesionalismo" en el servicio de habitaciones?, ¿Cuál es el grado de satisfacción de los huéspedes de los hospedajes (no clasificados) de la localidad Huancayo, en cuanto al atributo "cortesía" en el servicio de habitaciones?, ¿Cuál es el grado de satisfacción de los huéspedes de los hospedajes (no clasificados) de la localidad Huancayo, en cuanto al atributo "seguridad" en el servicio de 
habitaciones?, ¿Cuál es el grado de satisfacción de los huéspedes de los hospedajes (no clasificados) de la localidad Huancayo, en cuanto al atributo "credibilidad/accesibilidad" en el servicio de habitaciones?, ¿Cuál es el grado de satisfacción de los huéspedes de los hospedajes (no clasificados) de la localidad Huancayo, en cuanto al atributo "comprensión con el cliente" en el servicio de habitaciones?, ¿Cuál es el grado de satisfacción de los huéspedes de los hospedajes (no clasificados) de la localidad de Huancayo, en cuanto al atributo "accesibilidad al centro de hospedaje"?, ¿Cuál es grado de incidencia del los atributos: "tangible", "fiabilidad", "capacidad de respuesta", "profesionalismo", "cortesía", "seguridad", "credibilidad/accesibilidad" y "comprensión con el cliente" en la rentabilidad del cliente las empresas de hospedaje (no clasificados) de la localidad de Huancayo. El estudio de este problema ha permitido constatar que los niveles de satisfacción de clientes están relacionado con los niveles de fidelización de los mismos en las empresas de hospedaje (no clasificadas) de la localidad de Huancayo, esta relación es significativa al nivel del $5 \%$ y la intensidad de la asociación es de $63,6 \%$ que se tipifica como alta. Asimismo, ha precisado que la zona de ubicación de los centros de hospedaje constituye en un factor que determina los niveles de satisfacción de los clientes de las empresas de hospedaje. Del mismo modo, con respecto a las dimensiones de atributos tangibles, atributo fiabilidad, atributo capacidad de respuesta, atributo profesionalismo, atributo cortesía, atributo seguridad, atributo credibilidad, atributo comprensión con el cliente y accesibilidad al centro de hospedaje, como partes de la variable independiente las relaciones con el nivel de fidelización de los clientes han resultado muy significativas tanto a nivel general como según zona de ubicación de las empresas de hospedaje.

\section{OBJETIVOS}

\section{OBJETIVO GENERAL}

Determinar la incidencia de la satisfacción del cliente en la fidelización de clientes, en las empresas de hospedaje (no clasificadas) de la localidad de Huancayo.

\section{OBJETIVOS ESPECÍFICOS}

- $\quad$ Establecer el nivel de satisfacción de los huéspedes de los hospedajes (no clasificados) de la localidad Huancayo, en cuanto a los elementos tangibles del servicio de habitaciones.

- $\quad$ Establecer el grado de satisfacción de los huéspedes de los hospedajes (no clasificados) de la localidad Huancayo, en cuanto a los atributos "tangibles" del servicio de habitaciones.

- $\quad$ Establecer el grado de satisfacción de los huéspedes de los hospedajes (no clasificados) de la localidad Huancayo en cuanto al atributo "fiabilidad" en el servicio de habitaciones.

- Conocer el grado de satisfacción de los huéspedes de los hospedajes (no clasificados) de la localidad Huancayo, en cuanto al atributo "capacidad de respuesta" en el servicio de habitaciones.

- Conocer el grado de satisfacción de los huéspedes de los hospedajes (no clasificados) de la localidad Huancayo, en cuanto al atributo "profesionalismo" en el servicio de habitaciones.

- $\quad$ Establecer el grado de satisfacción de los huéspedes de los hospedajes (no clasificados) de la localidad Huancayo, en cuanto al atributo "cortesía" en el servicio de habitaciones.

Establecer el grado de satisfacción de los huéspedes de los hospedajes (no 
clasificados) de la localidad Huancayo, en cuanto al atributo "seguridad" en el servicio de habitaciones.

- $\quad$ Establecer el grado de satisfacción de los huéspedes de los hospedajes (no clasificados) de la localidad Huancayo, en cuanto al atributo "credibilidad/accesibilidad" en el servicio de habitaciones.

- $\quad$ Establecer el grado de satisfacción de los huéspedes de los hospedajes (no clasificados) de la localidad Huancayo, en cuanto al atributo "comprensión con el cliente" en el servicio de habitaciones.

- Determinar el grado de satisfacción de los huéspedes de los hospedajes (no clasificados) de la localidad de Huancayo, en cuanto al atributo "accesibilidad al centro de hospedaje" en el servicio de habitaciones.

- Conocer la incidencia de los atributos: "tangible", "fiabilidad", "capacidad de respuesta", "profesionalismo", "cortesía", "seguridad", "credibilidad / accesibilidad", "comprensión con el cliente" y "accesibilidad al centro de hospedaje" en la fidelización de clientes en las empresas de hospedaje (no clasificadas) de la localidad de Huancayo.

\section{METODOLOGÍA}

\section{TIPO DE INVESTIGACIÓN}

Por la naturaleza del estudio, la presente investigación es tipificada como una investigación aplicada.

\section{NIVEL DE INVESTIGACIÓN}

En cuanto la profundidad de los conocimientos a estudiar, la presente investigación es del nivel explicativo o causal.

\section{MÉTODO DE LAINVESTIGACIÓN}

Se ha utilizado el método científico.

\section{Métodos Específicos}

- Inductivo-Deductivo.

- Analítico-Sintético.

\section{DISEÑO DE LA INVESTIGACIÓN}

De acuerdo con Sierra Bravo, es el explicativo causal, cuyo esquema es:

Donde:

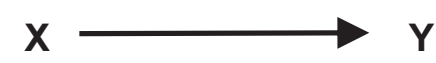

X: Satisfacción del cliente.

Y: Fidelización de clientes.

Z: Procedencia (V. Interviniente).

\section{POBLACIÓN, MUESTRAY MUESTREO}

\section{Población}

Así pues, en el presente trabajo de investigación se ha considerado como elementos de la población en estudio en un primer lugar a la zona de ubicación de las empresas hoteleras que está constituido por la zona residencial, comercial y especial; en segundo lugar los clientes que hacer uso del servicio de las mencionadas empresas hoteleras. Con la información proporcionada por la Municipalidad Provincial de Huancayo se han identificado 195 empresas hoteleras; asimismo en cuanto al flujo de usuarios que hacen uso del servicio de las empresas hoteleras, de la información del Ministerios de Comercio Exterior y Turismo se deduce que en promedio es de 523 usuarios.

\section{Muestra}

Cuadro para el cálculo del muestreo polietápico.

\begin{tabular}{lcccc}
\hline \hline \multirow{2}{*}{ Estrato } & \multicolumn{2}{c}{$\begin{array}{c}\text { Primera etapa } \\
\text { (establecimientos) }\end{array}$} & \multicolumn{2}{c}{$\begin{array}{c}\text { Segunda etapa } \\
\text { (usuarios) }\end{array}$} \\
\cline { 2 - 5 } & $\mathbf{N}$ & $\mathbf{n}$ & $\mathbf{N}$ & $\mathbf{n}$ \\
\hline Residencial & 32 & 11 & 85 & 45 \\
Comercial & 124 & 43 & 333 & 75 \\
Especial & 39 & 13 & 105 & 50 \\
\hline \multicolumn{1}{c}{ Total } & 195 & $\mathbf{6 7}$ & $\mathbf{5 2 3}$ & $\mathbf{1 7 0}$ \\
\hline \hline
\end{tabular}

\section{Método General}




\section{Muestreo}

Se utilizó la técnica de muestreo probabilístico bietápico.

\section{TÉCNICAS E INSTRUMENTOS DE}

\section{RECOLECCIÓN DE DATOS}

Cuadro para las técnicas e instrumentos

\begin{tabular}{ll}
\hline \multicolumn{1}{c}{ TECNICA } & \multicolumn{1}{c}{ INSTRUMENTO } \\
\hline Encuesta & Ficha de encuesta \\
Análisis documental & Guía de análisis documental \\
Entrevista & Ficha de entrevista \\
Econométrica & Cuestionario econométrico \\
\hline
\end{tabular}

\section{TÉCNICAS DE PROCESAMIENTO Y ANÁLISIS DEDATOS}

Para el análisis de la información obtenida mediante los instrumentos de medición se usaron básicamente dos técnicas: estadística descriptiva e inferencial.

\section{RESULTADOS Y DISCUSIÓN}

Cuadro para la relación de la satisfacción y fidelización del cliente de los hospedajes.

\begin{tabular}{|c|c|c|c|c|c|c|c|c|}
\hline \multirow{3}{*}{$\begin{array}{l}\text { Nivel de } \\
\text { satisfacción } \\
\text { del cliente }\end{array}$} & \multicolumn{6}{|c|}{ Nivel de tidelizacıón del cliente } & \multirow{2}{*}{\multicolumn{2}{|c|}{ Total }} \\
\hline & \multicolumn{2}{|c|}{ Bajo } & \multicolumn{2}{|c|}{ Medio } & \multicolumn{2}{|c|}{ Alto } & & \\
\hline & f & $\%$ & f & $\%$ & f & $\%$ & f & $\%$ \\
\hline Muy malo & 5 & 2,9 & - & - & - & - & 5 & 2,9 \\
\hline Malo & 14 & 8,2 & 2 & 1,2 & - & - & 16 & 9,4 \\
\hline Normal & 13 & 7,6 & 22 & 12,9 & 19 & 11,2 & 54 & 31,8 \\
\hline Bueno & 2 & 1,2 & 10 & 5,9 & 56 & 32,9 & 68 & 40,0 \\
\hline Muy bueno & - & - & 1 & 0,6 & 26 & 15,3 & 27 & 15,9 \\
\hline Total & 34 & 20,0 & 35 & 20,6 & 101 & 59,4 & 170 & 100,0 \\
\hline & $\vec{m}_{\mathrm{c}}$ & 5,28 & & $\mathbf{T}_{0 \mathrm{rn}}$ & & & & \\
\hline
\end{tabular}

-Sig $=0,000$ [significativa ai nivel de $90_{0,05}$ ]

\section{Fuente: Base de datos.}

Con lo cual decimos que se ha encontrado evidencia empírica para rechazar la hipótesis nula y por lo tanto aceptamos la hipótesis de investigación, es decir aceptamos el hecho de que: el nivel de satisfacción de los clientes, incide de manera positiva y significativa en el nivel de fidelización de clientes de las empresas de hospedaje de la localidad de Huancayo.

La fuerza de la relación hallada lo determinamos mediante el coeficiente de contingencia definido en la ecuación $N^{\circ} 05$, cuyo valor es de CC=63,6\%. Cuadro para la relación de la satisfacción y fidelización del cliente a nivel de dimensiones.

\begin{tabular}{|c|c|c|c|c|}
\hline \multirow{3}{*}{$\begin{array}{l}\text { Relación de la Fidelización del Cliente } \\
\text { versus... }\end{array}$} & \multicolumn{3}{|c|}{ Zona } & \multirow{3}{*}{$\begin{array}{l}\text { Total } \\
\text { Sig. }\end{array}$} \\
\hline & Especial & Comercial & Residencial & \\
\hline & Sig. & Sig. & Sig. & \\
\hline Atributos tangibles & $0,000^{*}$ & $0,000 *$ & $0,000^{*}$ & $0,000 *$ \\
\hline Atributo fiabilidad & $0,000^{*}$ & 0,005 . & $0,000^{*}$ & $0,000^{*}$ \\
\hline Atributo capacidad de respuesta & $0,020^{*}$ & 0,288 & 0,065 & 0,000 * \\
\hline Atributo profesionalismo & $0,002^{\star}$ & 0,004 * & $0,000^{*}$ & $0,000^{*}$ \\
\hline Atributo cortesia & $0.000 *$ & $0,029 *$ & $0,002^{*}$ & $0,000 *$ \\
\hline Atributo segurid ad & $0,001^{*}$ & $0,002^{*}$ & $0,000^{\wedge}$ & $0,000^{\circ}$ \\
\hline Atributo credibilidad & $0,000^{*}$ & $0,001 *$ & $0,000^{*}$ & $0,000 *$ \\
\hline Atributo com prensión con el cliente & $0,002^{\star}$ & $0,005^{*}$ & $0,000^{*}$ & $0,000^{\star}$ \\
\hline Accesibilidad al centro de hospedaje & $0,000^{*}$ & $0,001 *$ & $0,000^{*}$ & $0,000 *$ \\
\hline
\end{tabular}

Como se puede observar para cada una de las dimensiones el nivel de contraste de significancia obtenido a nivel total o general es de una relación altamente significativa Sig. $=0,000<0,05$ lo que indica que a nivel de componentes o dimensiones también las dos variables están relacionados de forma significativa, asimismo este resultado es compatible con la relación total. Queda así corroborado las nueve hipótesis específicas de investigación en el sentido de ser válidas.

Los mismos resultados se obtienen cuando se relacionan las variables a nivel de zona de ubicación de las empresas, siendo las dos únicas excepciones en el componente de atributo capacidad de respuesta, para las zonas comercial y residencial.

Cuadro para la relación de la satisfacción y fidelización del cliente a nivel de grado de instrucción y procedencia.

\begin{tabular}{llcccc}
\hline \hline Relación de la Satisfacción y la & \multicolumn{4}{c}{ Estadística } \\
\cline { 2 - 6 } Fidel ización del Cliente, a nivel de ... & Chi cuadrado & N & Significancia & Fuerza \\
\hline \multirow{2}{*}{ Grado de instrucción Primaria } & 9,00 & 9 & 0,061 & - \\
& Secundaria & 49,46 & 55 & $0,000 *$ & $68,8 \%$ \\
& Superior & 63,18 & 106 & $0,000^{*}$ & $61,1 \%$ \\
\hline \multirow{2}{*}{ Procedencia } & Lima & 37,38 & 48 & $0,000^{*}$ & $66,2 \%$ \\
& Provinicias & 39,52 & 68 & $0,000^{*}$ & $60,6 \%$ \\
\hline
\end{tabular}

- Sig el,05 [Significativa al nival do seo,05]

Fuente: Base de datos 
En la tabla se muestran los resultados de la relación entre las dos variables en estudio a nivel del grado de instrucción y la procedencia de los clientes. Usando la misma metodología del caso anterior, notamos que en todos los casos las relaciones resultan significativas, a excepción del nivel primario de instrucción de los clientes, lo cual implica que para los clientes cuyo nivel de instrucción es el nivel primario la relación entre el nivel de satisfacción y la fidelización es incierta.

\section{CONCLUSIÓNES}

1. Se ha constatado que los niveles de satisfacción están relacionado con los niveles de fidelización de los clientes de las empresas de hospedaje de la localidad de Huancayo, esta relación es significativa al nivel del $5 \%$ y la intensidad de la asociación es de $63,6 \%$ que se tipifica como alta.

2. En cuanto a los niveles de satisfacción de los usuarios de las empresas de hospedaje de la localidad de Huancayo, predominan los niveles bueno y normal con un $40 \%$ y $31,8 \%$ respectivamente. Sin embargo, al realizar los contrastes teniendo en cuenta la zona de ubicación de las empresas, se concluye que los niveles de satisfacción de la zona especial, residencial y comercial difieren de forma significativa al nivel de $5 \%$; es así que la zona de ubicación se constituye en un factor que determina los niveles de satisfacción de los clientes de las empresas de hospedaje.

3. La fidelización de los clientes está relacionada con el nivel de apreciación que los clientes tienen de la empresa; de este modo, el $59,4 \%$ tiene un nivel de fidelización alto. Cabe destacar que los niveles de fidelización de los clientes que hacen uso de las empresas de hospedaje de las zonas especial y comercial son indiferentes entre sí, pero muy diferentes a los dela zona residencial.

4. En las dimensiones de atributos tangibles, atributo fiabilidad, atributo capacidad de respuesta, atributo profesionalismo, atributo cortesía, atributo seguridad, atributo credibilidad, atributo comprensión con el cliente y accesibilidad al centro de hospedaje, como partes de la variable independiente las relaciones con el nivel de fidelización de los clientes han resultado muy significativas tanto a nivel general como según zona de ubicación de las empresas de hospedaje. Únicamente existen excepción en la dimensión de atributo capacidad de respuesta para la zona comercial y residencial.

\section{REFERENCIAS}

- $\quad$ AFUAH, A. (1999). La dinámica de la innovación organizacional, el nuevo concepto para lograr ventajas competitivas $y$ rentabilidad. Oxford University Press.

- $\quad$ aguilar J.A., CRESPO J.L., RUBIO M. (2007): "Análisis de la productividad y la eficiencia". Junta de Andalucía. Granada.

- $\quad$ ALBRETCH, Karl; RON, Semke. (2000). Gerencia del Servicio: Como hacer negocios en la nueva economía. Bogota. Tercera Edición.

- $\quad$ CABEZA, M. (2000). "La industria turística busca nuevos horizontes". Revista Economia № 16.

- $\quad$ COPI, I. (1995). Introducción a la Lógica. En (Eds.). Mexico D.F.. (Vol. 1, p. 699). (8a. Ed.). Mexico D.F., Mexico D.F., Mexico : Limusa.

- CARRASCO, S. (Eds.). (2006). Metodología de la Investigación Científica.( (2a. Ed.).). Lima, Lima, Perú: San Marcos. .

- $\quad$ GERSON, Richard F.(1999). "Como medir la satisfacción del cliente". Editorial Latinoamericana S.A.

Fecha de recepción: 05/04/2013

Fecha de aprobación: 08/04/2013

Correspondencia: Lorenzo Fidel Bernardo Sabuco 